\title{
The Development of DT-NB Hybrid Algorithms for Classifying Some Defective Dataset Types for Software Quality Prediction
}

\author{
Kattiya T. Yangyuen and Vichuda Rattanapian
}

\begin{abstract}
This research is the presenting the development of hybrid algorithms that is called DT-NB or Decision Tree Naïve Bayesian to predict about the software quality .Besides, it can develop the new technique of data mining for software industry or the current software engineering .Then, these techniques to make the comparison are Decision Tree, Rule-Based and Naïve Bayesian.Similarly, according to the analysis result of making the comparison for quality planning, it was found that the technique DT-NB had the correctness result of96 percent .Additionally, it was found that according to the analysis result of making comparison for quality assurance, the technique of DT-NB had the correctness result with76percent . On the same way, according to the result analysis of making comparison of quality control, it was found that the technique of DT-NB had the correctness result of92 percent .
\end{abstract}

Index Terms-Hybrid algorithms, software quality, business management.

\section{INTRODUCTION}

Nowadays, it has seen the difficulty of the software manufacturing management project obviously in the form of software or intangible product. However, the definitions of software including of software document can be seen clearly when comparing to the building project or the automobile manufacturing project as the tangible product. Thus, the software project executives can't notice for the clear effects.

In develop a hybrid algorithm to classify dataset type and predict software quality. previous work is prediction of current of any software should be full with good condition to call as "The quality software". and different is Better forecast by improved the process of Decision tree and Naïve Bayesian techniques to be suitable for digging data of NASA data set (KC2).

In addition, with these reasons the researcher has determined the hybrid data mining techniques between Decision Tree - Naïve Bayesian (DT-NB) for predicting the success of software management project in each organization. Similarly, it should check whether it has the attributes directly to the principle of software quality in your organization. This research question is Decision Tree - Naïve Bayesian (DT-NB). It can provide a good analysis of NASA data set (KC2) data. And analysis results of Quality Planning Information, Quality Assurance Information, and Quality Control Information found that the accuracy is at a good level,

Manuscript received May 30, 2019; revised November 23, 2019.

Kattiya T. Yangyuen is with the Department in information Technology of Rattana Bundit University, Thailand (e-mail: kattiya.kty@gmail.com).

Vichuda Rattanapian is with Rattana Bundit University, Thailand (e-mail: Rattanapian@gmail.com).
96, 92, 76 respectively.However, this research has the structures as this following: software quality and relevant researches, proposed methodology, experiment analysis and results, proof of DT-NB, and conclusion.

\section{SOFTWARE QuALITY}

Software quality is a measure of the quality of software that has been produced to meet production standards that meet international standards using CMMI criteria and project management maturity model in software quality measurement, [1] due as the quality of the software the research processes; namely, A. quality plan, B. quality assurance, C. quality control and D. Software Quality Management [2], [3].

\section{A. Quality Plan}

The document or several documents that together specify quality standards for practices, resources, specifications, and the sequence of activities relevant to a particular product, service and project or contract.The quality plans should define:

- objectives to be attained (for example, characteristics or specifications, uniformity, effectiveness, aesthetics, cycle time, cost, natural resources, utilization, yield, dependability, and soon).

- steps in the processes that constitute the operating practice or procedures of the organization.

- allocation of responsibilities, authority, and resources during the different phases of the process or project.

- specific documented standards, practices, procedures, and instructions to be applied.

- suitable testing, inspection, examination, and audit programs at appropriate stages.

- a documented procedure for changes and modifications to a quality plan as a process is improved.

- a method for measuring the achievement of the quality objectives.

- Other actions necessary to meet the objectives.

\section{B. Quality Assurance}

The management method to guarantee or ensure that a process or operation will achieve the desired quality results. However, the quality assurance is a management for effectiveness such as, ways to get the results you want every time or get the same result every time, depending on when the operation was successful. Second, if performed as usual every time without any complications, the consequences 
would be like that every time. The emphasis is to be carried out like this effective time. Is a way of ensuring quality. Finally, systematic management in order to ensure that the process or operation will achieve the specified quality results; it consists of a good analysis of the factors and characteristics of the factors. That will result in quality planning activities that will cause factors that look good on quality.Implementation of the activity plan quality inspection.

- create a software quality assurance plan.

- define roles and authority's responsibility of the software quality assurance team.

- advising and reviewing standard project operation plans and the development process that has been established for use in the project.

- review the work process in the project.according to the software quality assurance action plan.

- check software referring delivering to the system user / customer by referring to agreements or contracts.

- software analysis / evaluation summary to let the software development team know from time to time.

\section{Quality Control}

The plan generally includes detailed information on:

- an overview or introduction of the project or process detailing the background, need, scope, activities, and important dates or deadlines.

- the organizational structure or org chart detailing necessary team members, including external vendors.

- each team member's responsibilities and qualifications necessary to fulfill stated duties.

- work verification (e.g., who is responsible for carrying out a task, as well as who is responsible for checking the work).

- supplier standards (e.g., specify the standards the prospective suppliers must meet before they can bid on a contract, such as ISO 9001:2015).

- a list of qualified suppliers.

- testing parameter.

- performance standards and how performance will be documented.

- acceptance criteria.

- Deliverables.

- a feedback mechanism for internal and/or external customer feedback.

- quality control procedures.

- Audits.

- training(e.g., overview, job-specific, or refresher training).

- corrective action and preventive actions, including the person(s) responsible for CAPA.

- suggested corrective action.

- required notifications.

- any references or related materials, including performance ratings or performance report.

\section{Software Quality Management}

The software quality management is the identification of quality software features. Methods of measuring quality And the improvement of the quality of software development, including those obtained from each process

In addiction software quality management It is the application of quality management work for general product manufacturing industry to be used in software production in all aspects. Whether it is the process of software products And resources used by setting standards and working processes to meet those standards

identifying methods for defining quality software features quality measurement methods and improving the quality of the software development process.

identifying methods for defining quality software features and quality measurement methods and quality improvement and improving the quality of the software development process and including what is obtained from each process.

\section{RESEARCH TECHNIQUES}

The suggested data mining techniques, such as data classification or data segmentation In this process, many techniques will be used to get the best answer. Therefore, it may sometimes be necessary to go back to the Data Preparation step to convert some data to suit each technique. Examples of techniques for data analysis such as:

\section{A. Nä̈ve Bayesian}

The classification is a data mining classifier which is created by the Naïve Bayesian classification probability principle. Will be used to analyze Seems to be something that has never happened by speculating from what had happened before using Bayes theory to solve problems and can compare performance with Decision Tree and Neural Network techniques. However, Equations used to solve problems is

$$
P\left(C_{i} \mid x\right)=P\left(x \mid C_{i}\right) P\left(C_{i}\right) / p(x)
$$

Work Procedure in Naïve Bayesian .

Step 1 Count the total number of transactions and find the class that is the result .Class results will have only 2 answers.

Step 2, separate the number of results per Number of transactions using Rule 1 .

Step 3 Separate the non-answer class to find the probability ratio per Transaction .

Step 4 The commissioning of the desired data The master must not be in the table .

Step 5 Take the information from item 3 to the master of the result is based on Rule 2 .

\section{B. Decision Tree Learning}

The Tree of Decision (English: Learning the Tree of Decision) is sponsored by DR. DOME and DR. JA is a way to assess the difference of beliefs. (Discrete-value function) and the tree plan may have a set of trees) so humans can read and understand the decision of the tree. Although in the machine learning (Machine learning) The decision tree is a mathematical model that predicts the type of object that depends on the nature of the internal node of the tree. The branch shows the possible values of diversity. The blade shows the type of object. However, business management 
decisions are an early map that helps in making decisions. By showing the value of resources to use the investment risk and possible decision-making results to help make decisions in creating a popular risk management plan Decision trees are part of decision theory and graph theory. The era of decision making is one of the basic ways to dig information. However, Equations used to solve problems is

$$
E(S)=p_{\text {yes }} \log _{2}\left(p_{\text {yes }}\right)-p_{\text {no }} \log _{2}\left(p_{\text {no }}\right)
$$

When considering entropy, it can be seen that entropy is between 0 and 1, which is zero when all cases have only one result, such as yes or no. And will have more value when starting with more different values or in other words, entropy is more valuable if the data is not pure and will decide what the result will be when entropy is 0 .

\section{Decision Tree Building Algorithm}

In define the characteristics of good early variables.The A variable is a good starting variable only if the sample data )Example (is divided into sets .The number of sets according to the number of possible values for each case) Instance (In that set, there is only one value, and the average of the entropy of the data set that has been divided )partition (is the lowest .Calls for the expected value of the entropy reduction after the data is divided by $\mathrm{A}$ that the knowledge of $\mathrm{A}$ is defined.

\section{RELEVANT RESEARCHES}

Software Quality have means the level of software product to response with the requirement of the software users. [1] Moreover, the property of any software should be full with good condition to call as "The quality software". In addition, nowadays it has gathered the techniques of software engineer to apply for the software quality development. [2] Similarly, the structures and the supporting of software product consist of activity management, costs, planning, patterns, analysis, determination, designing, operation, testing and maintenance of software product. On the other hand, it has brought the science to apply as the basic theory for software structures because it is based on the software engineering to use particularly with controlling software and science method. Thus, the development of software measurement is comprising of the total activity times for gathering information [3], such as costs and estimation, effectiveness of manufacturing, measurements and patterns, information gathering, quality patterns and measurements, reliability patterns, effectiveness assessment and patterns, structures and complex measurements, ability assessment, management measurements and method assessment and tools. Furthermore, this research is presenting about the applying of Decision tree evolution in software engineering for the report pattern and errors in 0-5 commands of lines of code, measurements of McCabe, and basic measurements of Halstead and the counting of applied branch numbers. However, the definition of several patterns with other errors to be designed newly or for testing or for maintaining carefully as well as applying in other ways. What's more, the learning about Decision trees to apply with data mining and work learning with tools are depending on using the Decision tree for make conclusion on objectives in each list and notice about the ability to solve problems in the best way. Significantly, it involves with the additional explanation of classifying tree patterns or regression of tree to explain the Decision tree theory consisting of type classifying and field properties to classify the Decision theory types and Decision analysis. Therefore, the Decision tree is relatively to the graphs and Decision patterns including of the specification of importance on problems and the occurring chances, such as resources, costs and benefits. Then, these fields can be applied to create the plan to achieve the goals with all selective operations as this following: 1. Selection random from attribute nodes with all attribute sets 2 . Testing from selection of attribute random to gain the stable value 3.Random from selection tree leaf node by replacing with attribute nodes and 4. Random from selection of attribute nodes by replacing with Decision node.However, the application of theory to the field below the applied Decision tree in classifying the physical quality management by using the techniques of data mining in the form of Decision tree. [4] Then, it can select the result of $\mathrm{C} 4.5$ from making expectation of the data mining measurement to search for the problems, solve the physical problems, improve the physical quality and make expectation from the patients in daily of the hospital accurately. Then, with the classification of this information, it will be beneficial to the hospital for allocate the physical resources correctly and meanwhile the applied Rule-Based in classifying the category of financial news gathering from online and newspaper in group with positivity and negativity. [5] Then, it can calculate the value to find the reliability in overall from the news. Moreover, the results gained from analyzing the financial news will make the financial reliability with Rule-Based to result in the Means with 75.6 percent and addition the applied Naïve Bayesian to classify the news documents in groups. [6] Moreover, it can calculate the value to find the probability of words in each type of news contents and find the probability of each news group. Then, the result to be classified is depending on the news group selection with the most probability as the answer of the learning information sets and made the comparison of the techniques to information management for the losses ones in data mining of classification work. [7] Then, it can bring this technique to make comparison with Decision tree, Bayesian Naïve Bayes and KNN. Besides, it is the way to use the information from UCI to make the test. Similarly, the result of the test can indicate that if the information is the number, it can remove the better record. However, the information as text with adding question mark can make the better effectiveness of information classification.

\section{MATH FOR HYBRID MODEL By DT-NB}

After it has passed the procedure of preparation, selection and processing of information from PROMISE Software Engineering Repository: NASA data set (KC2) [8], the researcher has improvement the process model for the check accuracy and measuring performance of software quality. The procedure is as follows:

Step 1: The analysis in the development of quality software and check out the relationship rules of Decision Tree is one technique to estimate the discrete-value function .Then, it has 
comprised with the sets of regulations, such as if-then by creating the model of decision tree to select the attributes with the relationship of class at most .In addition, it can be the top tree )root node .(After that, it can find the next attribute continuously for gaining the relationship of attribute to measurement called as Information Gain)IG .(Thus, the equation will be available in written form below.

$$
\text { entropy }\left(c_{1}\right)=-p\left(c_{1}\right) \log p\left(c_{1}\right), p\left(c_{1}\right)
$$

Step 2: The analysis in the development of quality software and check out the relationship rules of Naïve Bayesian is the method to solve the problems with classification for making prediction of results and explain the Naïve Bayesian technique to gain the good and rapid result with simplicity and rapidness by using the equation as this following:

$$
P(H \mid X)=\frac{P(X \mid H) P(H)}{P(X)}
$$

Step 3: The measurement procedure and the comparing of effectiveness between the decision tree and Naïve Bayesian )DT-NB .(Then, the relationship rule of Decision Tree Naïve Bayesian )DT-NB (is the way to reduce the dimension of information .Therefore, from equations 3.2.1 and 3.2.2 can be rewritten as the following equation.

$$
D T-N B\left(t_{i}\right)=1 \sum_{i=0}^{N} \sum_{i=1}^{N}\left[P\left(t_{i}\right)\right]^{2}+\frac{\mathrm{P}(X \mid H) \mathrm{P}(H)}{\mathrm{P}(X)}
$$

\section{Proposed Methodology}

This research has applied 4 data mining techniques to data processing for making prediction of information. Besides, the using information from PROMISE Software Engineering Repository: NASA data set (KC2). [3] With these research processes; namely, A. Information selection, B. Preparation of Information Set, C. Data Processing.

\section{A. Information Selection}

Information Selection is the selection in the pattern of selecting column with quite full information. Moreover, in each column it should have the value for every row as the same one. Besides, the value in each column shouldn't be repetitive, but it should edit information to be correct and perfect. Thus, it should adjust information to be suitable for making decision and setup the information group to reduce the dispersing of information. Therefore, it can find the means to collect samples of questionnaires prior to find the Means to estimate the values of population or $\mu$. As the result, it should determine the Confidence Interval on the Mean: Variance known is $l \leq \mu \leq u$ When $l$ isLower limit anduisupper limit, we are called as"Two-sided Confidence interval" by finding the Median of populations inside limit from the solution is $P(L \leq \mu \leq U)=1-\alpha$.thus, In this case $\alpha$ isError risk or the value to refer to the risk from prediction the value of $\mu$ with error, the reliability value and the risk value are $100(1-\alpha) \%$ or called as the percent of reliability. Similarly, it has the value called as One-sided confidence interval with the solution is $1 \leq \mu$. So that, according to the above solution, we are called as lower-confidence interval with the value oflor called aslower limit with this following $\mu \leq u$ and according to the above solution, it is called asupper-confidence interval by the value of $u$ is the value of upper limit. Thus, the value $100(1-\alpha) \%$ is called as the reliability of parameter $\mathrm{m}$. When we refer to the theory of sampling distribution for mean, it has the median value of $m$ and the dispersing value equally to $\sigma^{2} / n$. Therefore, it has the value is $Z=\bar{x}-\mu / \sigma / \sqrt{n}$ When disperse value as Normal, the value $\alpha$ should be divided by 2 . So that, in the case of finding two-sided confidence interval from the figure, it can be concluded as this following:

$$
P\left\{-Z \sigma_{/ 2} \leq Z \leq Z \sigma / 2\right\}=1-\alpha
$$

When replace with the value $z$, it has the value as this following:

$$
P\left\{-Z \sigma_{/ 2} \leq \frac{\bar{x}-\mu}{\sigma / \sqrt{n}} \leq Z \sigma / 2\right\}=1-\alpha
$$

According to the setting up of the new solution, it has the value as this following:

$$
P\left\{\bar{x}-Z \sigma_{/ 2} \partial / \sqrt{n} \leq \mu \leq \bar{x}+Z \sigma_{/ 2} \partial / \sqrt{n}\right\}=1-\alpha
$$

\section{B. Preparation of Information Set}

Preparation of Information Set to practicing and testing is the preparation setup to gain the most perfect information which can bring the preparation information to divide into 2 groups that are the practicing set and testing set with these details:

Practicing Set: it can divide into 2 of 3 ratios of all information. Testing

Set: it can divide into 1 of 3 ratios of all information.

\section{Data Processing}

This program has been applied in this research that is the program MATLAB for predicting in the part of Decision Tree, Rule-Based, Naïve Bayesian and DT-NB. Moreover, the program MATLAB is the popular one to predicting the software for data mining, and it is accepted popularly for making data processing in both practicing set and testing set.

\section{RESULT}

The analysis of making comparison for the technique to present with the new one of Decision Tree, Rule based and Naïve Bayesian.Besides, it can make the comparison for the presented technique of DT-NB to find the accuracy and the correctness value with the result of data mining for the quality software management.

According to the explanation of Fig. 1, it has applied the analysis result to make comparison of the quality planning by using the technique of Decision Tree and Rule-Based. Besides, it was found that the technique of Rule-Based had the correctness result with 92 percent when comparing to the technique of Rule-Based with the correctness result of 89 
percent. In addition, for the technique of Naïve Bayesian and the technique of DT-NB, it was found that the technique of DT-NB had the correctness result with96 percent when comparing to the technique of Naïve Bayesian with the correctness result of 80 percent.

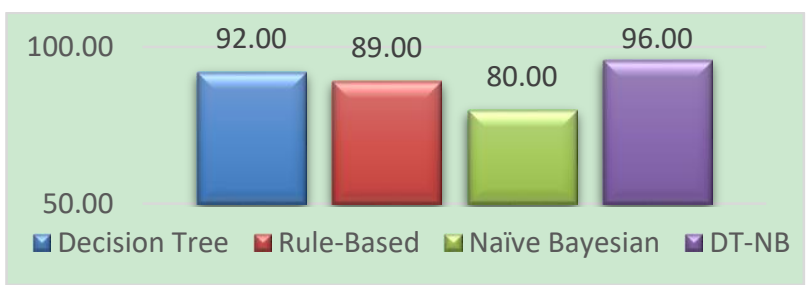

Fig. 1. Quality planning information.

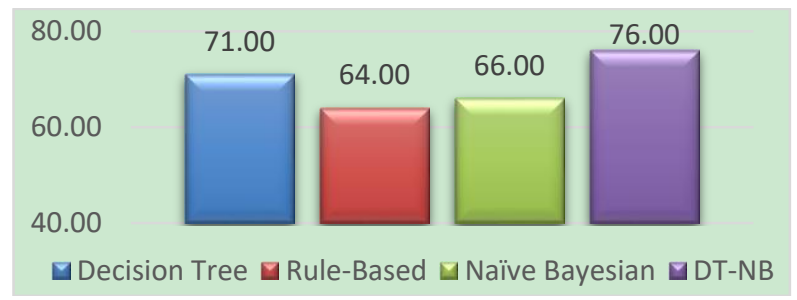

Fig. 2. Quality assurance information.

According to the explanation of Fig. 2, the analysis result to make comparison of quality assurance has applied the technique of Decision Tree and Rule-Based. Furthermore, it was found that the technique of Decision Tree had the correctness result of71 percent when comparing to the technique of Rule-Based with the correctness result of 64percent. Similarly, for the technique of Naïve Bayesian and DT-NB, it was found that with the technique DT-NB It has the correctness of 76 percent when comparing to the technique of Naïve Bayesian with the correctness result of66 percent.

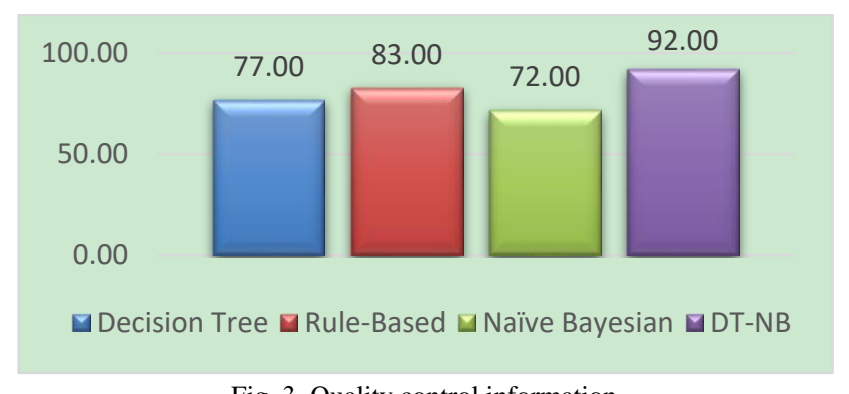

Fig. 3. Quality control information.

According to the explanation of Fig. 3, the analysis result to make comparison of quality control has applied the technique of Decision Tree and Rule-Based. Then, it was found that the technique of Decision Tree has the correctness result with77 percent when comparing to the technique of Rule-Based with the correctness result of 83 percent. Similarly, for the technique of Naïve Bayesian and DT-NB, it was found that the technique of DT-NB had the correctness result with92 percent when comparing to the technique of Naïve Bayesian with the correctness result with 72 percent.

\section{MATH FOR PROOF OF DT - NB}

If $\mathrm{L}$ is a regular language on the alphabet $\sum$ then there exists a right-linear grammar $G=\left(Q, \sum, \delta, q_{0}, F\right)$ such that $L=L(G)$. However, Proof: Let $\mathrm{M}=\left(\mathrm{Q}, \sum, \delta, q_{0}, F\right)$ be a dfa accepts L. we assume that. $Q=\left\{q_{0}, q_{1}, \ldots, q_{n}\right\}$ And $\sum=\left\{a_{0}, a_{1} \ldots, a_{n}\right\}$. Construct the right-linear grammar $\mathrm{G}=\left(V, \sum, S, P\right)$ with $V=\left\{a_{0}, a_{1} \ldots, a_{n}\right\}$. And $S=a_{0}$, for each transition.

$$
\delta\left(q_{i}, q_{j}\right)=q_{k}
$$

Of $M$, we put in $P$ the production.

$$
q_{i} \rightarrow q_{j}, q_{k}
$$

In addition, if $q_{k}$ is in $F$, we add to $\mathrm{P}$ the production.

$$
q_{k} \rightarrow \lambda
$$

We first show that $G$ defined in this way can generate every string in $L$, consider $\omega \in L$ of the form

$$
\omega=a_{i}, a_{j} \ldots a_{k}, a_{l}
$$

For $M$ to accept this string it must make moves via.

$$
\begin{gathered}
\delta\left(q_{0}, a_{1}\right)=q_{p}, \\
\delta\left(q_{p}, a_{j}\right)=q_{r}, \\
\vdots \\
\delta\left(q_{s}, a_{k}\right)=q_{t}, \\
\delta\left(q_{t}, a_{l}\right)=q_{f} \in F
\end{gathered}
$$

By construction, the grammar will have one production for each of these $\delta$ 's. therefore, we can make the derivation.

$$
\begin{aligned}
& q_{0} \Rightarrow a_{i} q_{p} \Rightarrow a_{i} a_{j} q_{r} \Rightarrow a_{i} a_{j} \ldots a_{k} a_{l}, \\
& \quad \Rightarrow a_{i} q_{j} \Rightarrow a_{k} a_{l} q_{f} \Rightarrow a_{i} a_{j} \ldots a_{k} a_{l},
\end{aligned}
$$

With the grammar $G$ and $\omega \in L(G)$. Conversely, If $\omega \in$ $L(G)$, then its derivation must have the form 2.3.

$$
\delta^{*}\left(q_{0}, a_{i}, a_{j} \ldots a_{k}, a_{l}\right)=q_{f}
$$

Completing the proof.

\section{CONCLUSION}

The analysis result of making comparison for software quality Information has applied the analysis for 3 techniques, such as the information set of quality planning with 4 attributes, the information set of quality assurance with 3 attributes and the information set of quality control with 3 attributes. Besides, it was found that the technique to give the accuracy value to predict the software quality value is called as DT-NB. Moreover, it is in the form of Hybrid algorithm between Decision Tree and Naïve Bayesian.In addition, according to the basic analysis, it was found that this technique can be predicted as the most accuracy way that is called Decision Tree and Naïve Bayesian. Thus, it can increase the accuracy to predict for the software engineering and increasing of dimensions; namely, symmetry matrix and skew symmetry matrix. Then, it should separate the testing set to be 2 sets that are the practicing set and the testing set to be analyzed the result for predicting for the software quality. 
However, this research is the presenting of algorithm hybrid techniques that is called DT-NB or Decision Tree - Naïve Bayesian to predict about the software quality. Besides, it can develop the new technique of data mining for software industry or the current software engineering. Then, these techniques to make the comparison are Decision Tree, Rule-Based and Naïve Bayesian.Similarly, according to the analysis result of making the comparison for quality planning, it was found that the technique DT-NB had the correctness result of 96 percent. Additionally, it was found that according to the analysis result of making comparison for quality assurance, the technique of DT-NB had the correctness result with76percent. On the same way, according to the result analysis of making comparison of quality control, it was found that the technique of DT-NB had the correctness result of92 percent. Then, the proving of the solution for DT-NB is the way to create the continuous working patterns in the form of regulations in the tree structure and it can gain the good results, easy understanding without complexity and rapidness to make prediction. Furthermore, the increasing of dimension for classification resulted in more accuracy values for attribute and class by using the symmetric matrix and the skew-symmetric matrix. Then, it has gained much information with the prediction of accuracy values and correctness values for attributes increasingly. Thus, with the proving of the solution for symmetric matrix, it can answer for the assumption about the prediction of the correctness and the accuracy values much better.

\section{CONFLICT OF INTEREST}

The authors declare that there are no conflicts of interests regarding the publication of this paper.

\section{AUTHOR CONTRIBUTIONS}

We help each other think the research and managing the share in the process. Firstly, Kattiya T. Yangyuen is responsible for the operation of searching for theoretical information and related research. Second, Vichuda Rattanapian has conducted the task of managing the information that is used in the test for further analysis. Finally, we helped to the continue writing articles for further publication.

\section{ACKNOWLEDGMENT}

This research is supported by some data by [Tim Menzies] from the information source on the website [PROMISE Software Engineering Repository]. We thank our colleagues from [RattanaBundit University] that provides insights and expertise that help research a lot and we thank 3 "anonymous" reviewers for their so-called insights.

\section{REFERENCES}

[1] C. J. Kent, Project Management Maturity Model, Second Edition, NW, pp. 3-181, 2007.

[2] H. Kerzner, Using the Project Management Maturity Model Strategic Planning for Project Management, Canada, pp. 41-142, 2005.

[3] N. E. Fenton and S. L. Pfleeger, "Software metrics, A rigorous \& practical approach," International Thomson Computer Press, London 1997, p. 638, 1997.

[4] L. J. Chen et al., "Research on prediction method for pivotal indicator of hospital medical quality using decision tree," China, pp. 247-250, 2015.
[5] T. L. Mi, P. W. San, and C. Kim, "Rule-based sentiment analysis for financial news," New Zealand, pp. 1601-1606, 2015.

[6] J. Supakpong and S. Nuanwan, "Hierarchical text classification: A restructured approach, Transactions on engineering, computing and technology," Thailand, pp. 24-29, 2004.

[7] N. Kerdprasop, "Comparative study of techniques to handle missing values in the classification task of data mining," Thailand, pp. 1-3, 2008.

[8] SERepository. [Online]. http://promise.site.uottawa.ca/SERepository/

Available:

Copyright $(2) 2020$ by the authors. This is an open access article distributed under the Creative Commons Attribution License which permits unrestricted use, distribution, and reproduction in any medium, provided the original work is properly cited (CC BY 4.0).

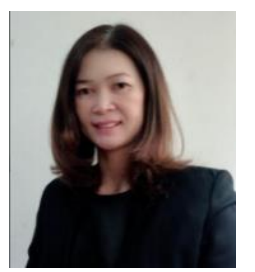

Kattiya Tawsopar Yangyuen was born at Bangkok, on January 19, 1974. The author's educational background is listed as follows: May 2005 - May 2012, the doctor of philosophy in information technology, University of Sripratum, Bangkok, Thailand. May 2000 - July 2003, the masters in telecommunication and computer, University of Rangsit, Bangkok, Thailand. May 1993 - May 1997, the bachelor of computer science, University of Rajabhat, Akhonratchasima, Thailand. May 1986 - May 1992, High School Certificate equivalent, Suranari Withaya High School, Bangkok, Thailand.

Kattiya's work experiences: Teacher, Bangkok Thailand (May 2003 until now) the head of Department in information Techonology of Rattana Bundit University, Bangkok, Thailand. Plblications: AMPM3 criteria of algorithm summation for classifying datamining of software quality management. Tokyo, Japan: ICDLE, 2018. Rough-Mutual Feature Selection Based-on Minimal-Boundary and Maximal- Lower. Chonburi, Thailand : BUU, 2016. Measure the maturity of risk management software. Bangkok, Thailand : TIME-iCON, 2016. Analyzing of Data Mining by Using Technique of Naïve Bayesian for Prediction of the Relationship Pattern to Manage with Software Risks. Singapore : ITECT, 2016. Current and previous research interests are in the areas of System Analysis and Design, Software Engineering, and Database System, e-Learning Technology.

Asst. Prof. Dr. Kattiya Tawsopar Yangyuen worked for Academic Technical Committee at ICDLE2018 in Japan. Academic Technical Committee ICDLE2017 in Spain. Academic Technical Committee ITECT2016 in Singapore.

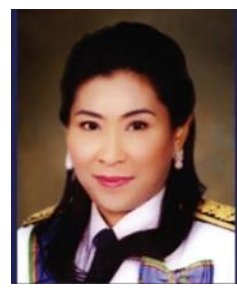

Vichuda Rattanapian was born at Bangkok, on May 5, 1963. The author's educational background is listedas follows: the B.Ed.(Honour) in French and English, Faculty of Education at Chulalongkorn University, Bangkok, Thailand in 1986; the M.Ed. in educational technology at Penn State University, Pennsylvania, U.S.A. in 1988; and the Ph.D. in educational technology at Penn State University, Pennsylvania, U.S.A. in 1992.

Vichuda's work experiences included Board Member at RattanaBundit University, Bangkok, Thailand since 2014 until present; a member of the Thai Senate from 2011 - 2014; the vice president of the Academic Affair at RattanaBundit University from 2006 - 2011; the lecturer in Faculty of Education at Chulalongkorn University from 1992 - 2006; the deputy director of Educational Technology Center at Chulalongkorn University from 1994 - 1998. Program enhance Self-efficacy Student Punyarat High School, Bangkok, Thailand, SSRU, 2018.

Lists of work for committees and publications are as follows. 1) The Senate Standing Committee and Sub Committee consisted of Chairman of the Monetary Finance Banking and Financial Institutions Standing Committee; Member of the Standing Committee on Foreign Affairs; Member of the Standing Committee on Education; Chairman of the ASEAN Community Readiness Following up Sub Committee; and Member of the Standing Committee on Sports. 2) The Inter-Parliamentary Union (IPU) included Member of the United Nations Affairs Standing Committee. 3) Parliamentary Friendship Groups consisted of Executive Committee of Thai - US Parliamentary Friendship Group; Executive Committee of Thai - US Parliamentary Friendship Group; Executive Committee of Thai - UK Parliamentary Friendship Group; Executive Committee of Thai - Estonia Parliamentary Friendship Group; and Executive Committee of Thai Sweden Parliamentary Friendship Group. 